\title{
Predicting ischemic stroke after carotid artery stenting based on proximal calcification and the jellyfish sign
}

\author{
Nobuhiko Ichinose, MD, ${ }^{1}$ Seiji Hama, MD, PhD, ${ }^{1}$ Toshio Tsuji, DrEng, ${ }^{2}$ Zu Soh, DrEng, ${ }^{2}$ \\ Hideaki Hayashi, DrEng, ${ }^{2}$ Yoshihiro Kiura, MD, PhD, ${ }^{1}$ Shigeyuki Sakamoto, MD, PhD, ${ }^{1}$ \\ Takahito Okazaki, MD, PhD, ${ }^{1}$ Daizo Ishii, MD, ${ }^{1}$ Katsuhiro Shinagawa, MD, PhD, ${ }^{1}$ and \\ Kaoru Kurisu, MD, PhD'
}

\begin{abstract}
1Department of Neurosurgery, Graduate School of Biomedical and Health Science; and ${ }^{2}$ Graduate School of Engineering, Hiroshima University, Hiroshima, Japan
\end{abstract}

\begin{abstract}
OBJECTIVE Carotid artery stenting (CAS) has been considered to prevent ischemic strokes caused by stenosis of the cervical carotid artery. The most common complication of CAS is new cerebral infarction. The authors have previously reported that the jellyfish sign-the rise and fall of the mobile component of the carotid plaque surface detected by carotid ultrasonography_suggests thinning and rupture of the fibrous cap over the unstable plaque content, such as the lipid-rich necrotic core or internal plaque hemorrhage. The authors' aim in the present study was to evaluate the risk of a new ischemic lesion after CAS by using many risk factors including calcification (size and location) and the jellyfish sign.

METHODS Eighty-six lesions (77 patients) were treated with CAS. The presence of ischemic stroke was determined using diffusion-weighted imaging (DWI). Risk factors included calcification of the plaque (classified into 5 groups for size and 3 groups for location) and the jellyfish sign, among others. Multiple linear regression analysis (stepwise analysis and partial least squares [PLS] analysis) was conducted, followed by a machine learning analysis using an artificial neural network (ANN) based on the log-linearized gaussian mixture network (LLGMN). The additive effects of the jellyfish sign and calcification on ischemic stroke after CAS were examined using the Kruskal-Wallis test, followed by the Steel-Dwass test.
\end{abstract}

RESULTS The stepwise analysis selected the jellyfish sign, proximal calcification (proximal Ca), low-density lipoprotein (LDL) cholesterol, and patient age for the prediction model to predict new DWI lesions. The PLS analysis revealed the same top 3 variables (jellyfish sign, proximal $\mathrm{Ca}$, and LDL cholesterol) according to the variable importance in projection scores. The ANN was then used, showing that these 3 variables remained. The accuracy of the ANN improved; areas under the receiver operating characteristic curves of the stepwise analysis, the PLS analysis, and the ANN were 0.719, 0.727 , and 0.768 , respectively. The combination of the jellyfish sign and proximal Ca indicates a significantly increased risk for ischemic stroke after CAS.

CONCLUSIONS The jellyfish sign, proximal Ca, and LDL cholesterol were considered to be important predictors for new DWI lesions after CAS. These 3 factors can be easily determined during a standard clinical visit. Thus, these 3 variables-especially the jellyfish sign and proximal $\mathrm{Ca}$-may be useful for reducing the ischemic stroke risk in patients with stenosis of the cervical carotid artery.

https://thejns.org/doi/abs/10.3171/2017.1.JNS162379

KEY WORDS ischemic stroke; carotid artery stenting; artificial neural network; carotid artery plaque; calcification; jellyfish sign; diffusion-weighted imaging; vascular disorders

ABBREVIATIONS ANN = artificial neural network; $\mathrm{AUC}=$ area under the ROC curve; $\mathrm{BMI}=$ body mass index; $\mathrm{Ca}=$ calcification; $\mathrm{CAS}=$ carotid artery stenting; $\mathrm{CEA}=$ carotid endarterectomy; DWI = diffusion-weighted imaging; LDL = low-density lipoprotein; LLGMN = log-linearized gaussian mixture network; LOSOCV = leave-one-subjectout cross-validation; NASCET = North American Symptomatic Carotid Endarterectomy Trial; PLS = partial least squares; PRESS = predicted residual error sum of squares; $\mathrm{ROC}=$ receiver operating characteristic; $\mathrm{VIP}=$ variable importance in projection; $\mathrm{WBC}=$ white blood cell.

SUBMITTED September 22, 2016. ACCEPTED January 19, 2017.

INCLUDE WHEN CITING Published online July 7, 2017; DOI: 10.3171/2017.1.JNS162379. 
$\mathrm{S}$ TENOSIS of the cervical carotid artery is a common cause of ischemic strokes, including transient ischemic attacks and cerebral infarction. Therefore, prophylactic surgeries such as carotid endarterectomy (CEA) or carotid artery stenting (CAS) have been considered to prevent ischemic strokes. The extensive multicenter, prospective, randomized controlled Stenting and Angioplasty with Protection in Patients at High Risk for Endarterectomy (SAPPHIRE) trial ${ }^{28}$ and the Carotid Revascularization Endarterectomy Versus Stenting Trial (CREST) ${ }^{19}$ demonstrated no significant difference in stroke prevention between CAS and CEA. The former procedure is thought to be less invasive ${ }^{15,31}$ and has emerged as an alternative to CEA for the treatment of symptomatic or asymptomatic stenosis of the cervical carotid artery. 5,31

The most common complication of CAS is new infarction detected by diffusion-weighted imaging (DWI) of high-intensity lesions (new DWI lesions), with an incidence of $37 \%(9 \%-70 \%)$ following CAS versus $10 \%$ (0\%-27\%) after CEA. ${ }^{25}$ Deployment of the stent expands the stenosed lumen, but the radial force placed on the carotid artery wall by this expansion can collapse the plaque and push it into the lumen. ${ }^{24}$ During stent deployment, the intraplaque contents (lipid, thrombotic, and calcified) slip through the stent strut and cause cerebral infarction (new DWI lesions). Thus, to prevent new DWI lesions, attention should be paid to the characteristics of the intraplaque content, especially its vulnerability in terms of intraplaque hemorrhage, thin or ruptured fibrous cap, and lipid-rich necrotic core. ${ }^{3}$ On the other hand, the relationship of calcification to the risk of ischemic stroke remains a matter of controversy; some reports on large series of CAS cases agree that calcification is related to the risk of ischemic stroke, ${ }^{12,26}$ but other reports disagree., ${ }^{9,20}$ A major confounder in these trials is the lack of a uniform definition of lesion calcification.

We previously reported that carotid ultrasonography can detect the mobile component of the carotid plaque surface, which rises and falls in a manner asynchronous with arterial pulsatile wall motion (jellyfish sign) ${ }^{16}$ In that study, our pathological findings demonstrated that fibrous cap thickness negatively correlates with ultrasonographic plaque surface movement in the jellyfish-positive portion of the plaque, suggesting that the jellyfish sign indicates thinning and rupture of the fibrous cap over the unstable plaque content, that is, the lipid-rich necrotic core or internal plaque hemorrhage. Thinning and rupture of the fibrous cap would lead to the release of thrombogenic factors into the arterial lumen, resulting in repeated ischemic stroke during a short interval after diagnosis. Thus, the jellyfish sign is listed in the glossary of the Japan Society of Ultrasonics in Medicine and will be listed in the next Japanese guidelines on ultrasonography of the cervical vascular system by the Japan Academy of Neurosonology. Several reports indicate that the jellyfish sign is a risk for ischemic stroke, ${ }^{7,14,21}$ but no study including the jellyfish sign has evaluated the risk factors of CAS.

To prevent new DWI lesions after CAS, technological developments have been incorporated into the CAS procedure. We previously described a useful CAS procedure for decreasing distal embolization by intraplaque contents even with vulnerable plaque, in which we provided dual protection via simultaneous flow reversal and a distal filter combined with blood aspiration, with a low rate of adverse events; the rate of new DWI lesions was $17.3 \%$ and that of major adverse events (major stroke, myocardial infarction, or death) within 30 days of CAS was $1.05 \%{ }^{23}$ Despite this procedure, however, asymptomatic new DWI lesions were detected after CAS. Accurately predicting new DWI lesions after CAS may identify high-risk patients for the CAS procedure, thereby reducing morbidity. However, patient clinical parameters are highly complex, and machine learning algorithms have been proposed as a means of identifying patients at high risk for poor outcomes since these systems have the advantage of easily incorporating newly available data to improve prediction performance., ${ }^{1,30}$

An artificial neural network (ANN), a kind of machine learning method, is a computing system made up of a number of simple, highly interconnected processing elements, constituting a nonlinear discriminant analysis. ${ }^{11}$ Today, ANNs have been applied in various fields because of their usefulness for pattern recognition and classification tasks. They learn complex interactions among inputs and identify relationships in input data that may not be apparent from analysis done by humans..$^{13}$ General ANNs, however, need a large sample size of training data to determine the network structure. In particular, the log-linearized gaussian mixture network (LLGMN) proposed by Tsuji et al. has been widely used for pattern classification problems of various bioelectric signals. ${ }^{27,29}$ An ANN based on the LLGMN can estimate a posteriori probability. One of the merits of using the LLGMN is that identification precision remains high even if there are few learning data.

Our aim in the present study was to evaluate the risk of a new ischemic lesion after CAS using several risk factors including calcification (size and location) and the jellyfish sign. We first evaluated variables best able to predict new ischemic lesions using multiple linear regression analysis with stepwise analysis and partial least squares (PLS) regression analysis. We then examined the validity of these variables with machine learning using an ANN based on the LLGMN.

\section{Methods \\ Patients}

This study was approved by our institutional review committee, and written informed consent was obtained from all patients. In the period from April 2012 to December 2015, a total of 117 consecutive lesions in 104 patients (94 males and 10 females; mean age 77 years, range 50-89 years) with carotid artery stenosis were treated with CAS (114 lesions, 101 patients) and CEA (3 lesions, 3 patients) at the Hiroshima University neurosurgical department. We performed CAS as a first-choice surgical treatment for carotid artery stenosis; however, 3 patients elected to undergo CEA. For the present study, we selected CAS cases in which the same procedure of dual protection combined with blood aspiration via the femoral arterial approach was performed. There were 36 symptomatic cases (41.9\%) at admission. Exclusion criteria comprised the following: use of the brachial arterial approach (4 lesions), lack of 
radiological imaging for evaluation (23 lesions), and restenosis (1 lesion; in total, 28 lesions).

\section{Risk Factors}

We assessed the described vascular risk factors based on the following. Body mass index (BMI) was calculated from patient height and weight on admission (weight [kg]/ height $^{2}[\mathrm{~m}]$ ). Symptomatic carotid artery stenosis was defined as focal neurological symptoms that were sudden in onset and referable to the appropriate carotid artery distribution, including one or more transient ischemic attacks characterized by focal neurological dysfunction or transient monocular blindness, or one or more minor ischemic strokes. The definition was contingent on the occurrence of carotid symptoms within the previous 6 months. The Brinkman smoking index ${ }^{4}$ is the number of cigarettes smoked per day multiplied by the number of years of smoking. Hypertension was defined as a history of using antihypertensive agents, systolic blood pressure > $140 \mathrm{~mm} \mathrm{Hg}$, or diastolic pressure $>90 \mathrm{~mm} \mathrm{Hg}$ after the first ischemic stroke event or at the first clinic attendance. Dyslipidemia was defined as using statins or having serum low-density lipoprotein (LDL) cholesterol $\geq 140 \mathrm{mg}$ / dl. Diabetes mellitus was defined as using oral hypoglycemic agents or insulin, and a glycosylated hemoglobin level $\geq 6.5 \%$ (National Glycohemoglobin Standardization Program).

\section{Imaging Technique}

Magnetic resonance imaging studies at field strengths of $3.0 \mathrm{~T}$ were performed 1 day before and 1 day after the CAS procedure. To increase the accuracy of our evaluation, we determined new ischemic lesions as regions of high intensity on DWI with both a usual b-value of 1000 and a more sensitive b-value of 4000. A tandem lesion was defined as showing stenosis $(>50 \%)$ at both the cervical and intracranial carotid arteries on the same side as the original stenosis with MR angiography.

To confirm permanent ischemic lesions, repeat followup using MRI (T1-weighted, T2-weighted, and FLAIR) was performed in 27 of the 36 cases with new DWI lesions more than 3 months after CAS. The MR images were interpreted by consensus between 2 experienced researchers blinded to patient information, including data on calcification and the jellyfish sign.

Calcification of the plaque was detected with CT scanning. To clarify the definition of calcification (Ca), we characterized 3 groups according to location (narrowest $\mathrm{Ca}$, narrowest portion; distal $\mathrm{Ca}, 1 \mathrm{~cm}$ distal from narrowest portion; and proximal $\mathrm{Ca}, 1 \mathrm{~cm}$ proximal from narrowest portion) and 5 groups according to size $(0$, none; $1,<1 / 4$ of the outer perimeter; $2,1 / 4$ to $1 / 2 ; 3,1 / 2$ to $3 / 4$; $4,>3 / 4)$. The CT images were interpreted by consensus between 2 experienced researchers blinded to patient information.

We detected the mobile component (jellyfish sign and floating plaque) and ulceration using ultrasonography: LOGIQ 7 system (GE Yokogawa Medical Systems) with a 3- to $10-\mathrm{MHz}$ broadband linear array transducer. The jellyfish sign of the carotid artery plaque, defined as the presence of mobile components that rose and fell in a manner inconsistent with arterial pulsatile wall motion, was evaluated under high-resolution B-mode ultrasonography. ${ }^{16}$ Examinations were performed by an experienced ultrasonographer.

Stenosis rate was calculated according to the North American Symptomatic Carotid Endarterectomy Trial (NASCET) method using digital subtraction angiography. ${ }^{6}$

We also divided proximal Ca into 2 groups (-, absent; + , present). Then we classified patients into 4 groups according to the presence or absence of the jellyfish sign and proximal $\mathrm{Ca}(\mathrm{J}-\mathrm{Ca}-$, jellyfish sign-/proximal $\mathrm{Ca}-; \mathrm{J}-\mathrm{Ca}+$, jellyfish sign-/proximal $\mathrm{Ca}+$; J+Ca-, jellyfish sign+/proximal $\mathrm{Ca}-; \mathrm{J}+\mathrm{Ca}+$, jellyfish sign+/proximal $\mathrm{Ca}+$ ).

\section{Medication}

Antiplatelet drugs (75 mg clopidogrel, $100 \mathrm{mg}$ aspirin, and/or $200 \mathrm{mg}$ cilostazol) were administered for at least 1 week before CAS. The same neurointerventionist performed CAS with patients under local anesthesia, and an activated clotting time $>275$ seconds was maintained using heparin during the procedure. After the CAS procedure, argatroban was administered via continuous intravenous infusion for 12 hours. Dual antiplatelet drugs were administered for 3 months, and a single antiplatelet drug was prescribed indefinitely.

\section{Interventional Protocol}

In this study, we performed CAS with the dual protection of a distal filter and simultaneous flow reversal combined with blood aspiration, as described previously. ${ }^{23} \mathrm{Fig}$ ure 1 shows representative angiographies and a schema. Briefly, sheaths were placed into both the femoral artery and femoral vein. The common carotid artery and external carotid artery were occluded with a balloon guide catheter and a wire balloon system, respectively. A filter wire system was placed in the distal side of the stenosed lesion. An external arteriovenous shunt line, made by connecting the proximal end of the guide catheter and the sheath of the femoral vein, generated reversed flow at the stenosed portion (flow reversal) because of the difference between the arterial and venous pressure. Under the dual protection of a distal filter and simultaneous flow reversal, we performed predilatation first, followed by stenting and then postdilatation. We aspirated blood through the aspiration catheter as it was moved from the stent to the distal filter, and then the protection devices were removed.

\section{Statistical Analysis}

We used JMP software (version 10.0, SAS Institute Inc.) for linear analyses. Variables considered to be continuous values for entry in the prediction models (counted number of new DWI lesions after CAS) included patient age, sex, BMI, symptomatic status, Brinkman smoking index, lesion side, hypertension, dyslipidemia, diabetes, atrial fibrillation, white blood cell (WBC) count, hemoglobin level, platelet count, C-reactive protein level, high-density lipoprotein cholesterol, LDL cholesterol, triglycerides, hemoglobin A1c, estimated glomerular filtration rate, ulcer, 

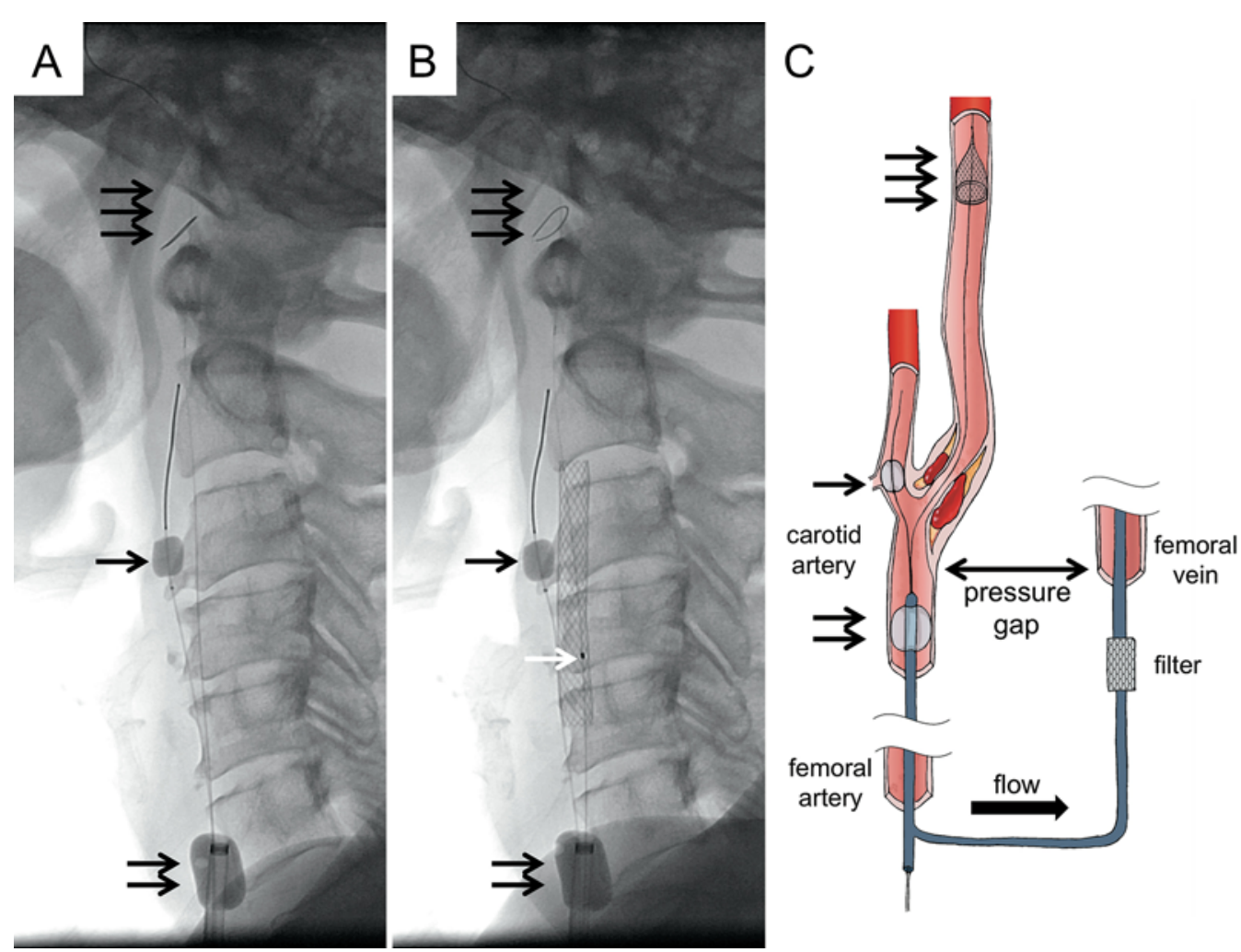

FIG. 1. A: Lateral unsubtracted angiographic view during CAS utilizing flow reversal, a distal filter, and blood aspiration. The external carotid artery (ECA; single black arrow) and common carotid artery (CCA; double black arrows) were occluded with balloons. A distal filter (triple black arrows) was deployed into the high cervical internal carotid artery (ICA). B: After stent deployment, blood was manually aspirated through the aspiration catheter (single white arrow) several times as it was moved from the stent to the distal filter. C: A schema of flow reversal due to the pressure gap between the femoral vein and collateral flow (bold black arrow) from the ICA. The internal filter of the arteriovenous shunt circuit was set to catch debris from the carotid artery plaque. Figure is available in color online only.

jellyfish sign, floating plaque, tandem lesion, proximal $\mathrm{Ca}$, narrowest $\mathrm{Ca}$, distal $\mathrm{Ca}$, and stenosis rate according to NASCET.

To test the correlation with the counted number of new DWI lesions, the least squares method (fitting the regression line) was used to compare continuous variables and the Wilcoxon signed-rank test was used to compare categorical variables. We identified variables to be retained in the analyses by a stepwise multiple linear regression procedure with a stopping rule of the $\mathrm{p}$ value threshold. The $\mathrm{p}$ value for variables to enter and stay in the prediction models was set at 0.1 .

A PLS analysis was performed to find combinations of the predictors that had a large covariance with the response values, solving the multicollinearity problem caused by many variables. We retained the smallest number of latent factors for which the predicted residual error sum of squares (PRESS) was minimum. The importance of each predictor in the construction of the PLS was assessed from the variable importance in projection (VIP) scores of Wold.

Supervised machine learning was then attempted using the LLGMN. In the experiment, we first prepared binary labels according to the presence of DWI lesions. Given the training data with the binary labels as the teacher vector, the LLGMN was trained using Newton's method with a learning rate of $1.0 .^{2}$ The classification accuracy was then evaluated based on leave-one-subject-out cross-validation (LOSOCV). The number of input variables was set referring to the VIP score of the former PLS analysis, and the number of gaussian components for each class was set as 1. In addition, confusion matrices were also calculated, allowing better representation of the performance of the LLGMN. The algorithm was programmed using $\mathrm{C}++$ and the Eigen (http://eigen.tuxfamily.org).

We then evaluated the capability of the predictive variables to discriminate the number of new DWI lesions by constructing the receiver operating characteristic (ROC) curve and by calculating each area under the ROC curve (AUC) in the stepwise analysis, the PLS analysis, and the ANN based on the LLGMN analysis.

Finally, the counted number of new DWI lesions in each of the 4 groups generated according to the presence or absence of the jellyfish sign and calcification $(\mathrm{J}-\mathrm{C}-$, $\mathrm{J}-\mathrm{C}+, \mathrm{J}+\mathrm{C}-$, and $\mathrm{J}+\mathrm{C}+$ ) was compared using the KruskalWallis test, followed by the Steel-Dwass test, with JMP.

\section{Results}

\section{Baseline Characteristics Among All Patients}

Table 1 shows the characteristics of the 86 lesions (77 patients) included in this study. Symptomatic cases includ- 
TABLE 1. Characteristics of 86 CAS cases

\begin{tabular}{|c|c|}
\hline Variable & No. \\
\hline Age in yrs & $71.5 \pm 6.78$ \\
\hline Male sex & $69(89.6)$ \\
\hline $\mathrm{BMI}$ in $\mathrm{kg} / \mathrm{m}^{2}$ & $23.6 \pm 2.59$ \\
\hline Rt lesion side & $36(41.9)$ \\
\hline Symptomatic & $36(41.9)$ \\
\hline Brinkman smoking index & $667 \pm 559$ \\
\hline \multicolumn{2}{|l|}{ Risk factors } \\
\hline Hypertension & $64(74.4)$ \\
\hline Dyslipidemia & $66(76.7)$ \\
\hline Diabetes & $33(38.4)$ \\
\hline Atrial fibrillation & $6(6.98)$ \\
\hline \multicolumn{2}{|l|}{ Blood exam } \\
\hline WBC in $\times 10^{3} / \mu \mathrm{l}$ & $5.94 \pm 1.51$ \\
\hline $\mathrm{Hb}$ in $\mathrm{g} / \mathrm{dl}$ & $13.2 \pm 1.32$ \\
\hline PLT in $\times 10^{3} / \mu \mathrm{l}$ & $208 \pm 121$ \\
\hline CRP in mg/dl & $0.128 \pm 0.219$ \\
\hline HDL cholesterol in mg/dl & $52.9 \pm 15.6$ \\
\hline LDL cholesterol in mg/dl & $81.2 \pm 23.2$ \\
\hline $\mathrm{TG}$ in mg/dl & $131 \pm 67.7$ \\
\hline $\mathrm{HbA1c}$ in $\%$ & $6.36 \pm 0.791$ \\
\hline eGFR in $\mathrm{ml} / \mathrm{min} / 1.73 \mathrm{~m}^{2}$ & $64.8 \pm 15.9$ \\
\hline \multicolumn{2}{|l|}{ Ultrasonography } \\
\hline Ulcer & $37(43)$ \\
\hline Jellyfish sign & $26(30.2)$ \\
\hline Floating plaque & $8(9.3)$ \\
\hline \multicolumn{2}{|l|}{ Radiological findings } \\
\hline New DWI lesion & $36(41.9)$ \\
\hline No. of lesions & $1.31 \pm 2.47$ \\
\hline Tandem lesion & $14(16.3)$ \\
\hline \multicolumn{2}{|l|}{ Proximal Ca score } \\
\hline $1-2$ & $42(48.8)$ \\
\hline $3-4$ & $6(6.98)$ \\
\hline \multicolumn{2}{|l|}{ Narrowest Ca score } \\
\hline $1-2$ & $39(45.3)$ \\
\hline $3-4$ & $18(20.9)$ \\
\hline \multicolumn{2}{|l|}{ Distal Ca score } \\
\hline $1-2$ & $17(20)$ \\
\hline $3-4$ & $0(0)$ \\
\hline Stenosis rate per NASCET method & $67.6 \pm 22.8$ \\
\hline
\end{tabular}

$\mathrm{CRP}=\mathrm{C}$-reactive protein; Distal $\mathrm{Ca}=$ calcification $1 \mathrm{~cm}$ distal from the narrowest part; eGFR = estimated glomerular filtration rate; $\mathrm{Hb}=$ hemoglobin; $\mathrm{HbA1C}$ $=$ hemoglobin A1c; $\mathrm{HDL}=$ high-density lipoprotein; Narrowest $\mathrm{Ca}=$ calcification at the narrowest part; PLT = platelet; Proximal $\mathrm{Ca}=$ calcification $1 \mathrm{~cm}$ proximal from the narrowest part; $T G=$ triglyceride.

Values expressed as mean \pm standard deviation or number of cases (\%).

ed $14(53.8 \%)$ of the 26 jellyfish-positive cases versus 22 (36.7\%) of the 60 jellyfish-negative cases.

\section{Clinical Outcomes}

The overall technical success rate was $100 \%$, and all stenoses were dilated. New hyperintense areas were detected on postoperative DWI in 36 cases (41.9\%). There were no major adverse events within 30 days of CAS. Minor stroke with slight finger dexterity movement disorder was seen in 1 patient $(1.3 \%)$, and motor aphasia was seen in 1 patient (1.3\%); these patients recovered within several days. Transient ischemic attacks were seen in 6 cases (6.98\%; paresis 2 cases, paresthesia 1 case, motor aphasia 2 cases, paresis and motor aphasia 1 case). One patient presented with paresthesia of the fingers without new DWI lesions.

Among the 36 cases with new DWI lesions, follow-up MRI was performed in 27 cases (92 lesions) more than 3 months after CAS, and 18 lesions (19.6\%) in 11 cases $(40.7 \%)$ were detected as permanent ischemic change.

\section{Bivariate Analysis}

Bivariate analysis between every factor and the number of the new DWI lesions revealed 3 variables that were positively significantly associated with new DWI lesions (Table 2): jellyfish sign, proximal Ca, and LDL cholester$\mathrm{ol}$. The $\mathrm{p}$ values for these 3 variables were $0.0002,0.0003$, and 0.0427 , respectively.

\section{Multiple Regression Analysis \\ Stepwise Analysis}

Four variables-jellyfish sign, proximal $\mathrm{Ca}, \mathrm{LDL}$ cholesterol, and patient age-were entered and remained in the stepwise multiple linear regression analysis (Table 3). The coefficient of determination $\left(\mathrm{R}^{2}\right)$ of this model was 0.357 with $\mathrm{p}<0.0001$. The variance inflation factors for each variable were $<2.0$, indicating minimal collinearity.

\section{Partial Least Squares Analysis}

In the PLS procedure, the PRESS was minimum (0.976) when the latent factor was 1 . The coefficient of determination $\left(\mathrm{R}^{2}\right)$ of this model was 0.391 , with $\mathrm{p}<0.0001$. The VIP scores are listed in Fig. 2, demonstrating that the jellyfish sign and proximal Ca had the highest VIP scores, followed by LDL cholesterol.

\section{Artificial Neural Network Analysis Based on the LLGMN}

Three variables-jellyfish sign, proximal Ca, and LDL cholesterol-were kept by removing variables one by one from all 30 variables in the order of ascending priorities of the VIP scores of the former PLS analysis. The confusion matrix based on the LOSOCV using these 3 variables showed that accuracy was $68.6 \%$, with a sensitivity of 0.5 and specificity of 0.82 (Table 4).

\section{Comparison of AUC}

Figure 3 shows ROC curves between the predicted and actual presence of a new DWI lesion. The AUCs for the stepwise analysis, the PLS analysis, and the ANN were $0.719,0.727$, and 0.768 , respectively.

\section{Correlation Between the Jellyfish Sign or Proximal $\mathrm{Ca}$ and New DWI Lesion}

The presence of the jellyfish sign and proximal Ca was 
TABLE 2. Bivariate analysis between every factor and new DWI lesions

\begin{tabular}{|c|c|c|}
\hline Variable & p Value & $\begin{array}{l}\text { Correlation } \\
\text { Coefficient }\end{array}$ \\
\hline Age & 0.0795 & 0.0691 \\
\hline Sex & 0.0726 & \\
\hline BMI & 0.57 & 0.0594 \\
\hline Symptomatic & 0.634 & \\
\hline Brinkman smoking index & 0.321 & 0.00048 \\
\hline Lesion side & 0.0526 & \\
\hline \multicolumn{3}{|l|}{ Risk factors } \\
\hline Hypertension & 0.894 & \\
\hline Dyslipidemia & 0.663 & \\
\hline Diabetes & 0.996 & \\
\hline Atrial fibrillation & 0.464 & \\
\hline \multicolumn{3}{|l|}{ Blood exam } \\
\hline WBC & 0.329 & 0.175 \\
\hline $\mathrm{Hb}$ & 0.224 & 0.249 \\
\hline PLT & 0.333 & -0.00216 \\
\hline CRP & 0.835 & -0.258 \\
\hline HDL cholesterol & 0.866 & -0.00292 \\
\hline LDL cholesterol & $0.0427^{*}$ & 0.0234 \\
\hline TG & 0.431 & 0.00315 \\
\hline $\mathrm{HbA1c}$ & 0.164 & 0.474 \\
\hline eGFR & 0.597 & -0.00899 \\
\hline \multicolumn{3}{|l|}{ Ultrasonography } \\
\hline Ulcer & 0.571 & \\
\hline Jellyfish sign & $0.0002^{*}$ & \\
\hline Floating plaque & 0.653 & \\
\hline \multicolumn{3}{|l|}{ Radiological findings } \\
\hline Tandem lesion & 0.684 & \\
\hline Proximal Ca & $0.0003^{*}$ & 0.993 \\
\hline Narrowest Ca & 0.217 & 0.262 \\
\hline Distal Ca & 0.312 & -0.583 \\
\hline Stenosis rate by NASCET method & 0.0782 & -0.0207 \\
\hline
\end{tabular}

To test the correlation with the number of new DWI lesions, the least squares method (fitting the regression line) was used to compare continuous variables and the Wilcoxon signed-rank test was used to compare categorical variables. ${ }^{*} p<0.05$. thought to predict ischemic stroke after CAS. To clarify the additive effects of the jellyfish sign and proximal $\mathrm{Ca}$ on ischemic stroke after CAS, we compared the counted number of new DWI lesions among all 4 groups $(\mathrm{J}-\mathrm{C}-$, $\mathrm{J}-\mathrm{C}+$, $\mathrm{J}+\mathrm{C}-$, and $\mathrm{J}+\mathrm{C}+$ ) using the Kruskal-Wallis test, followed by the Steel-Dwass test (Fig. 4). The counted number of new DWI lesions tended to increase in the order of J-C-, $\mathrm{J}-\mathrm{C}+, \mathrm{J}+\mathrm{C}-$, and $\mathrm{J}+\mathrm{C}+(\mathrm{p}=0.0002)$. The Steel-Dwass test revealed a tendency for $\mathrm{J}-\mathrm{C}-$ and $\mathrm{J}+\mathrm{C}+(\mathrm{p}<0.0001)$, and $\mathrm{J}-\mathrm{C}+$ and $\mathrm{J}+\mathrm{C}+(\mathrm{p}=0.024)$ to differ.

\section{Discussion}

In the present study, we aimed to predict the likelihood of new DWI lesions after CAS by using multiple regression analysis (stepwise analysis and PLS analysis), followed by the use of the ANN based on the LLGMN. In a stepwise analysis, the jellyfish sign, proximal Ca, LDL cholesterol, and patient age were revealed in the prediction model to predict new DWI lesions. A PLS analysis revealed the same top 3 variables (jellyfish sign, proximal Ca, and LDL cholesterol) according to the VIP scores. We then used the ANN, which showed that these 3 variables remained. The accuracy rate of the ANN improved; AUCs of the stepwise analysis, the PLS analysis, and the ANN were $0.719,0.727$, and 0.768 , respectively. The machine learning method using the ANN based on the LLGMN was useful for predicting the risk factors for new DWI lesions after CAS. The jellyfish sign and proximal Ca showed additive effects on ischemic stroke after CAS per the Kruskal-Wallis test, followed by the Steel-Dwass test. Thus, the jellyfish sign, proximal $\mathrm{Ca}$, and LDL cholesterol are considered to be important predictors for new DWI lesions after CAS.

Ischemic lesions after CAS have been reported to be caused by an embolus from vulnerable atherosclerotic plaque, that is, plaque comprising intraplaque hemorrhage, a lipid-rich necrotic core, and a thin or ruptured fibrous cap. ${ }^{10}$ From our previous study, we determined that the jellyfish sign indicates thinning and rupture of the fibrous cap over the unstable plaque contents. ${ }^{16}$ Once the thinning fibrous cap ruptures, blood flow into the plaque may push the thrombogenic contents (that is, lipid, thrombus, and calcification) into the arterial lumen through the rupture site, resulting in ischemic stroke. Larger intraplaque lipid pools, which become a more extensive amount of thrombogenic contents when pushed into the arterial lumen, could increase the risk for new ischemic lesions. ${ }^{17}$ Thus, results in our present study indicate that a thinning and/or ruptured fibrous cap (jellyfish sign) over a larger lipid pool

TABLE 3. Stepwise multiple linear regression analysis predicting new DWI lesions

\begin{tabular}{lllllc}
\hline \multicolumn{1}{c}{ Variable } & Regression Coefficient & SD & $95 \% \mathrm{Cl}$ & VIF & $\mathrm{p}$ Value \\
\hline Jellyfish sign & 2.12 & 0.48 & 1.16 to 3.08 & 1.00 & $<0.0001$ \\
\hline Proximal Ca & 0.869 & 0.234 & 0.403 to 1.34 & 1.03 & 0.0004 \\
\hline LDL cholesterol & 0.0199 & 0.00959 & 0.000774 to 0.0389 & 1.02 & 0.0416 \\
\hline Patient age & 0.0608 & 0.0327 & -0.00421 to 0.126 & 1.02 & 0.0664 \\
\hline
\end{tabular}

$\mathrm{VIF}=$ variance inflation factors

Variables entered into the model comprised those featured in Table 2. This table shows the final step of analysis. The intercept was -6.04 . Adjusted coefficient of determination $\left(R^{2}\right)$ was 0.357 . The variance inflation factors for each variable were $<2.0$, indicating minimal collinearity. 


\begin{tabular}{lc|c|c} 
Variable & VIP & correlation coefficient \\
\hline Jellyfish sign & 2.575 & 0.242 \\
Proximal-Ca & 2.481 & 0.233 \\
LDL & 1.42 & 0.133 \\
Stenosis rate by NASCET & 1.237 & -0.116 \\
Age & 1.232 & 0.116 \\
HbAlc & 0.982 & & 0.092 \\
Af & 0.97 & & 0.091 \\
Lesion side (left) & 0.969 & & 0.091 \\
Sex(male) & 0.878 & & 0.082 \\
Narrowest-Ca & 0.872 & & 0.082 \\
Hb & 0.859 & & 0.081 \\
Floating plaque & 0.785 & & 0.074 \\
Distal-Ca & 0.714 & & -0.067 \\
Smoking Brinkman Index & 0.702 & & 0.066 \\
WBC & 0.691 & & 0.065 \\
PLT & & -0.064 \\
TG & 0.684 & & 0.052 \\
Dyslipidemia & 0.558 & & 0.049 \\
Ulcer & 0.525 & & 0.048 \\
Hypertension & 0.516 & & 0.039 \\
BMI & 0.412 & & 0.038 \\
eGFR & 0.402 & & -0.035 \\
Symptomatic & 0.375 & & -0.025 \\
Diabetes & 0.266 & & 0.016 \\
CRP & 0.165 & & -0.014 \\
HDL & 0.148 & & -0.011 \\
Tandem lesion & 0.119 & & \\
& 0.115 & & \\
\hline
\end{tabular}

FIG. 2. Variable importance in projection (VIP) scores estimate the importance of each variable in the projection used in a PLS model and are often used for variable selection. A variable with a VIP score $>0.8$ can be considered important in a given model. $\mathrm{AF}=$ atrial fibrillation; $\mathrm{CRP}=$ $\mathrm{C}$-reactive protein; Distal $\mathrm{Ca}=$ calcification $1 \mathrm{~cm}$ distal from the narrowest part; eGFR = estimated glomerular filtration rate; $\mathrm{Hb}=$ hemoglobin; $\mathrm{HbA1c}=$ hemoglobin A1c; $\mathrm{HDL}=$ high-density lipoprotein; Narrowest $\mathrm{Ca}$ = calcification at the narrowest part; $\mathrm{PLT}=$ platelet; Proximal $\mathrm{Ca}=$ calcification $1 \mathrm{~cm}$ proximal from the narrowest part; $\mathrm{TG}=$ triglyceride.

(high LDL cholesterol level) represents a high risk factor for new DWI lesions after CAS.

In this study, proximal $\mathrm{Ca}$ also indicated a higher risk for new DWI lesions after CAS. During deployment, a stent expands from distally to proximally; therefore, the soft content of the plaque also shifts from the distal to the
TABLE 4. Confusion matrix

\begin{tabular}{ccc}
\hline & \multicolumn{2}{c}{ LLGMN Outcome } \\
\cline { 2 - 3 } True Condition & Negative & Positive \\
\hline Negative & 44 & 6 \\
\hline Positive & 19 & 17 \\
\hline
\end{tabular}

proximal side. This mechanism is the so-called snowplow effect. ${ }^{22}$ When the soft content of the plaque is sandwiched between the expanded stent and the calcified component that makes plaque less elastic, the inner pressure of the plaque increases. A previously described flow-plaque interaction model has shown high flow stress concentrations at the proximal shoulder regions of the plaque, which lead to gradual fibrous cap thinning, indicating a high risk for plaque rupture. ${ }^{18}$ With the calcified component at the proximal shoulder portion of the fibrous cap (that is, the thinning and weak part), soft plaque would apply higher pressure, thereby squeezing the soft content of the plaque into the lumen. This is consistent with our current results showing that the combination of the jellyfish sign and proximal $\mathrm{Ca}$ (not just the jellyfish sign or just proximal $\mathrm{Ca}$ ) indicated a significantly increased risk for ischemic stroke after CAS. Thus, proximal Ca together with the jellyfish sign was thought to play a pivotal role in the development of new DWI lesions after CAS.

We previously reported that adding a distal filter and blood aspiration to flow reversal during CAS could provide effective distal embolic protection, despite the fact that there is a high incidence of debris from vulnerable plaques during CAS. Thus, we performed CAS as the first-choice surgical treatment for carotid artery stenosis. However, the percentage of patients with new DWI lesions postprocedure was high (41.9\%). This is because the detection of new DWI lesions using b-values of both 1000 and 4000 in this study was more accurate than using a single bvalue of 1000 , as has been done in previous studies. Moreover, permanent ischemic change was detected with repeat MRI at the late follow-up in $41 \%$ of cases with postprocedure DWI lesions (approximately 17\% of total cases). The correlation between DWI signal abnormality and ischemic
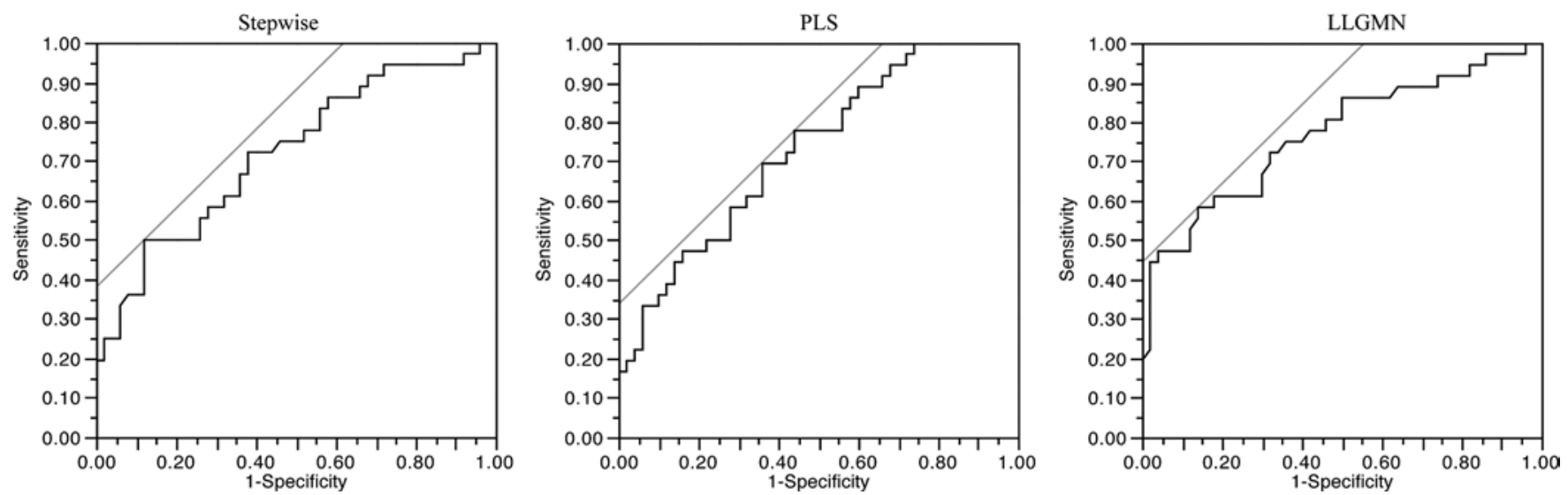

FIG. 3. Receiver operating characteristic curves between predicted and actual presence of a new DWI lesion for evaluating the capability of the predictive variables to discriminate the presence of new DWI lesions. The AUCs in the stepwise analysis, the PLS analysis, and the ANN based on the LLGMN analysis are presented. 


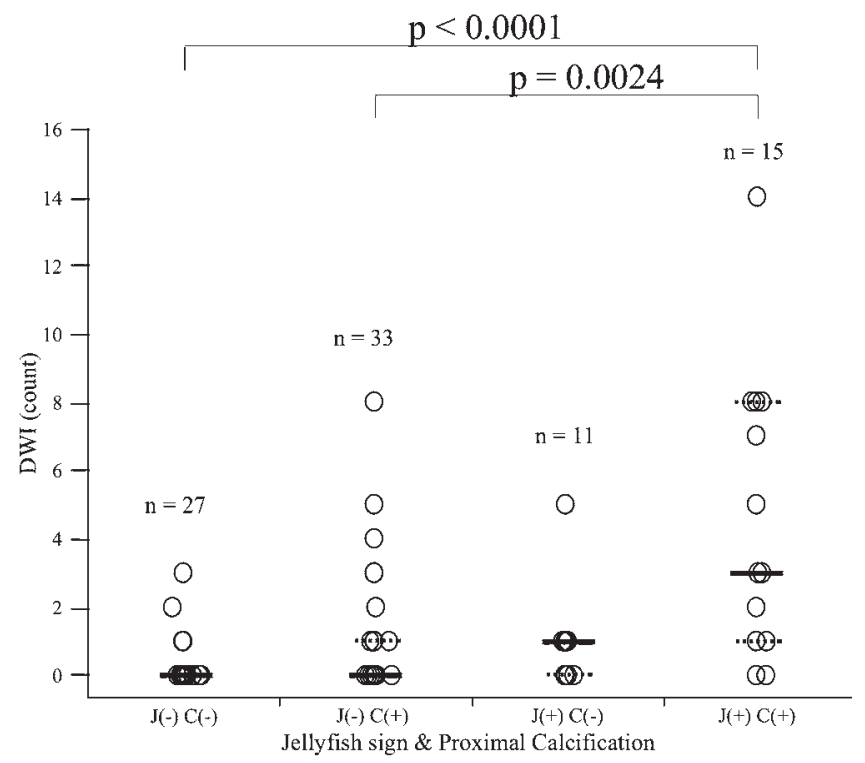

FIG. 4. Comparison of the counted number of new DWI lesions among all 4 groups according to the presence or absence of the jellyfish sign or proximal $\mathrm{Ca}$ (see text for definitions of groups). The Kruskal-Wallis test of the 4 subgroups showed a greater likelihood for new DWI lesions as you move from left to right on the $x$-axis. The Steel-Dwass test also indicated that these parameters can distinguish between some of the subgroups. Thick line indicates the median value, and the dotted line indicates the 25th and 75th percentiles within each category.

infarction (symptomatic vs asymptomatic) is controversial. A previous study demonstrated that patients with "silent" DWI lesions present with a significant decrease in global cognitive performance 6 months after $\mathrm{CAS} ;{ }^{8}$ thus, we should aim to strictly reduce the occurrence of DWI lesions after CAS. The 3 variables (jellyfish sign, proximal $\mathrm{Ca}$, and LDL cholesterol) in the present study with which one might predict new DWI lesions after CAS can be examined through typical ultrasonography, CT scanning, and blood tests, which can be easily performed during a standard clinical visit. Thus, these 3 variables, but especially the jellyfish sign and proximal $\mathrm{Ca}$, may be useful for reducing the ischemic stroke risk in patients with stenosis of the cervical carotid artery.

\section{Study Limitations}

Several methodological limitations of this study should be acknowledged. Firstly, detecting the jellyfish sign is not difficult for an experienced ultrasonographer, although we must always remember that ultrasonographic examination is observer dependent. Therefore, we have been trying to develop an ultrasonographic detection method with which anyone could detect the jellyfish sign. Secondly, there is still room for improvement in the AUC of the ANN used in this study. Thirdly, the number of patients in this study is relatively small. Additional studies including other potential factors and another machine learning algorithm can be conducted with a larger sample size for improvement.

\section{Conclusions}

In this study, the risk factors of the jellyfish sign, proxi- mal $\mathrm{Ca}$, and LDL cholesterol were important predictors for new DWI lesions after CAS. These 3 factors can be easily determined during a standard clinical visit. Thus, these 3 variables, but especially the jellyfish sign and proximal $\mathrm{Ca}$, may be useful for reducing the ischemic stroke risk in patients with stenosis of the cervical carotid artery.

\section{Acknowledgments}

This study was supported by the Ryokufu-kai Foundation.

\section{References}

1. Alpaydin E: Introduction to Machine Learning, ed 2. Cambridge, MA: MIT Press, 2010

2. Bennett AA: Newton's method in general analysis. Proc Natl Acad Sci U S A 2:592-598, 1916

3. Brinjikji W, Huston J III, Rabinstein AA, Kim GM, Lerman A, Lanzino G: Contemporary carotid imaging: from degree of stenosis to plaque vulnerability. J Neurosurg 124:27-42, 2016

4. Brinkman GL, Coates EO Jr: The effect of bronchitis, smoking, and occupation on ventilation. Am Rev Respir Dis 87:684-693, 1963

5. Dumont TM, Rughani AI: National trends in carotid artery revascularization surgery. J Neurosurg 116:1251-1257, 2012

6. Ferguson GG, Eliasziw M, Barr HW, Clagett GP, Barnes RW, Wallace MC, et al: The North American Symptomatic Carotid Endarterectomy Trial: surgical results in 1415 patients. Stroke 30:1751-1758, 1999

7. Funaki T, Iihara K, Miyamoto S, Nagatsuka K, Hishikawa T, Ishibashi-Ueda $\mathrm{H}$ : Histologic characterization of mobile and nonmobile carotid plaques detected with ultrasound imaging. J Vasc Surg 53:977-983, 2011

8. Gensicke H, Zumbrunn T, Jongen LM, Nederkoorn PJ, Macdonald S, Gaines PA, et al: Characteristics of ischemic brain lesions after stenting or endarterectomy for symptomatic carotid artery stenosis: results from the international carotid stenting study-magnetic resonance imaging substudy. Stroke 44:80-86, 2013

9. Gray WA, Yadav JS, Verta P, Scicli A, Fairman R, Wholey $M$, et al: The CAPTURE registry: predictors of outcomes in carotid artery stenting with embolic protection for high surgical risk patients in the early post-approval setting. Catheter Cardiovasc Interv 70:1025-1033, 2007

10. Gupta A, Baradaran H, Schweitzer AD, Kamel H, Pandya A, Delgado D, et al: Carotid plaque MRI and stroke risk: a systematic review and meta-analysis. Stroke 44:3071-3077, 2013

11. Hecht-Nielsen R: Neurocomputing. Boston: Addison-Wesley Publishing Co, 1990

12. Hofmann R, Niessner A, Kypta A, Steinwender C, Kammler J, Kerschner K, et al: Risk score for peri-interventional complications of carotid artery stenting. Stroke 37:2557-2561, 2006

13. Itchhaporia D, Snow PB, Almassy RJ, Oetgen WJ: Artificial neural networks: current status in cardiovascular medicine. $\mathbf{J}$ Am Coll Cardiol 28:515-521, 1996

14. Kakehi Y, Yamane F, Uemiya N, Tsukagoshi E, Niimi J, Neki $\mathrm{H}$, et al: A case of severe carotid artery stenosis with jellyfish sign performed endovascular therapy after statin treatment. J Neuroendovasc Ther 9:289-296, 2015

15. Kassaian SE, Goodarzynejad H: Carotid artery stenting, endarterectomy, or medical treatment alone: the debate is not over. J Tehran Heart Cent 6:1-13, 2011

16. Kume S, Hama S, Yamane K, Wada S, Nishida T, Kurisu K: Vulnerable carotid arterial plaque causing repeated ischemic stroke can be detected with B-mode ultrasonography as a mobile component: jellyfish sign. Neurosurg Rev 33:419430,2010 
17. Laloux P, Brucher JM: Lacunar infarctions due to cholesterol emboli. Stroke 22:1440-1444, 1991

18. Li ZY, Howarth SP, Tang T, Gillard JH: How critical is fibrous cap thickness to carotid plaque stability? A flow-plaque interaction model. Stroke 37:1195-1199, 2006

19. Mantese VA, Timaran CH, Chiu D, Begg RJ, Brott TG: The Carotid Revascularization Endarterectomy versus Stenting Trial (CREST): stenting versus carotid endarterectomy for carotid disease. Stroke 41 (10 Suppl):S31-S34, 2010

20. Mathur A, Roubin GS, Iyer SS, Piamsonboon C, Liu MW, Gomez CR, et al: Predictors of stroke complicating carotid artery stenting. Circulation 97:1239-1245, 1998

21. Moncayo KE, Vidal JJ, García R, Pereira D: Surgical management of a mobile floating carotid plaque. Interact Cardiovasc Thorac Surg 20:443-444, 2015

22. Oesterle SN: Angioplasty techniques for stenoses involving coronary artery bifurcations. Am J Cardiol 61:29G-32G, 1988

23. Sakamoto S, Kiura Y, Okazaki T, Shinagawa K, Ichinose N, Shibukawa M, et al: Usefulness of dual protection combined with blood aspiration for distal embolic protection during carotid artery stenting. Acta Neurochir (Wien) 157:371-377, 2015

24. Schillinger M, Gschwendtner M, Reimers B, Trenkler J, Stockx L, Mair J, et al: Does carotid stent cell design matter? Stroke 39:905-909, 2008

25. Schnaudigel S, Gröschel K, Pilgram SM, Kastrup A: New brain lesions after carotid stenting versus carotid endarterectomy: a systematic review of the literature. Stroke 39:19111919,2008

26. Setacci C, Chisci E, Setacci F, Iacoponi F, de Donato G, Rossi A: Siena carotid artery stenting score: a risk modelling study for individual patients. Stroke 41:1259-1265, 2010

27. Shima K, Tsuji T, Kandori A, Yokoe M, Sakoda S: Measurement and evaluation of finger tapping movements using log-linearized Gaussian mixture networks. Sensors (Basel) 9:2187-2201, 2009

28. Thomas DJ: Protected carotid artery stenting versus endar- terectomy in high-risk patients reflections from SAPPHIRE. Stroke 36:912-913, 2005

29. Tsuji T, Fukuda O, Ichinobe H, Kaneko M: A log-linearized Gaussian mixture network and its application to EEG pattern classification. IEEE Trans Syst Man Cybern C 29:60-72, 1999

30. Warwick K: March of the Machines: The Breakthrough in Artificial Intelligence. Urbana, IL: University of Illinois Press, 2004

31. Yadav JS, Wholey MH, Kuntz RE, Fayad P, Katzen BT, Mishkel GJ, et al: Protected carotid-artery stenting versus endarterectomy in high-risk patients. N Engl J Med 351:1493-1501, 2004

\section{Disclosures}

The authors report no conflict of interest concerning the materials or methods used in this study or the findings specified in this paper.

\section{Author Contributions}

Conception and design: Ichinose, Hama, Tsuji. Acquisition of data: Ichinose, Kiura, Sakamoto, Okazaki, Ishii, Shinagawa. Analysis and interpretation of data: Ichinose, Hama. Drafting the article: Ichinose. Critically revising the article: Ichinose, Hama. Reviewed submitted version of manuscript: all authors. Approved the final version of the manuscript on behalf of all authors: Ichinose. Statistical analysis: Ichinose, Hama, Tsuji, Soh, Hayashi. Study supervision: Hama, Kurisu.

\section{Correspondence}

Nobuhiko Ichinose, Department of Neurosurgery, Graduate School of Biomedical and Health Science, Hiroshima University, Kasumi 1-2-3, Minami-ku, Hiroshima-city, Hiroshima 734-8551, Japan. email: n.1s.stream@gmail.com. 